\title{
Randomization of Pulse Phases for Unambiguous and Robust Quantum Sensing
}

\author{
Zhen-Yu Wang, , $^{*}$ Jacob E. Lang, ${ }^{2}$ Simon Schmitt, ${ }^{3}$ Johannes Lang, ${ }^{3}$ Jorge Casanova, ${ }^{4,5}$ Liam McGuinness, ${ }^{3}$ \\ Tania S. Monteiro, ${ }^{2}$ Fedor Jelezko, ${ }^{3}$ and Martin B. Plenio ${ }^{1}$ \\ ${ }^{1}$ Institut für Theoretische Physik und IQST, Universität Ulm, Albert-Einstein-Allee 11, D-89069 Ulm, Germany \\ ${ }^{2}$ Department of Physics and Astronomy, University College London, Gower Street, London, England WC1E 6BT, United Kingdom \\ ${ }^{3}$ Institute of Quantum Optics, Universität Ulm, Albert-Einstein-Allee 11, D-89069 Ulm, Germany \\ ${ }^{4}$ Department of Physical Chemistry, University of the Basque Country UPV/EHU, Apartado 644, 48080 Bilbao, Spain \\ ${ }^{5}$ Ikerbasque, Basque Foundation for Science, Maria Diaz de Haro 3, 48013 Bilbao, Spain
}

(Received 5 March 2019; published 24 May 2019)

\begin{abstract}
We develop theoretically and demonstrate experimentally a universal dynamical decoupling method for robust quantum sensing with unambiguous signal identification. Our method uses randomization of control pulses to simultaneously suppress two types of errors in the measured spectra that would otherwise lead to false signal identification. These are spurious responses due to finite-width $\pi$ pulses, as well as signal distortion caused by $\pi$ pulse imperfections. For the cases of nanoscale nuclear-spin sensing and ac magnetometry, we benchmark the performance of the protocol with a single nitrogen vacancy center in diamond against widely used nonrandomized pulse sequences. Our method is general and can be combined with existing multipulse quantum sensing sequences to enhance their performance.
\end{abstract}

DOI: 10.1103/PhysRevLett.122.200403

Introduction.-The nitrogen-vacancy (NV) center [1] in diamond has demonstrated excellent sensitivity and nanoscale resolution in a range of quantum sensing experiments [2-5]. In particular, under dynamical decoupling (DD) control [6], the NV center can be protected against environmental noise [7-9] while at the same time being made sensitive to an ac magnetic field of a particular frequency [10]. This makes the NV center a highly promising probe for nanoscale nuclear magnetic resonance (NMR) and magnetic resonance imaging (MRI) [11-19]. Moreover, NV centers under DD control can be used to detect, identify, and control nearby single nuclear spins [20-28] and spin clusters [29-33] for applications in quantum sensing [34], quantum information processing [35,36], quantum simulations [37], and quantum networks [38,39].

Errors in the DD control pulses are unavoidable in experiments and limit performance, especially for a larger number of pulses. To compensate for detuning and amplitude errors in control pulses, robust DD sequences that include several pulse phases [7,40-42] were developed. However, these robust sequences still require good pulsephase control and, more importantly, they introduce a spurious harmonic response [43] due to the finite length of the control pulses. This spurious response leads to false signal identification (e.g., the misidentification of ${ }^{13} \mathrm{C}$ nuclei for ${ }^{1} \mathrm{H}$ nuclei), and hence negatively impacts the reliability and reproducibility of quantum sensing experiments. Under special circumstances, it is possible to control some of these spurious peaks [44-46]. However, it is highly desirable to design a systematic and reliable method to suppress any spurious response and to improve the robustness of all existing DD sequences, such as the routinely used $X Y$ family of sequences [40], the universally robust (UR) sequences [42], and other DD sequences leading to enhanced nuclear selectivity $[41,47]$.

In this Letter, we demonstrate that phase randomization upon repetition of a basic pulse unit of DD sequences is a generic tool that improves their robustness and eliminates a spurious response while maintaining the desired signal. This is achieved by, first, adding a global phase to the applied $\pi$ pulses within one elemental unit and, second, randomly changing this phase each time the unit is repeated. Our method is universal; that is, it can be directly incorporated to arbitrary DD sequences and is applicable for any physical realization of a qubit sensor.

DD-based quantum sensing.-We consider the interaction of the general form $\hat{H}^{\prime}(t)=\frac{1}{2} \hat{\sigma}_{z} \hat{E}(t)$ between a sensor qubit and its environment. Here, $\hat{\sigma}_{z}=|0\rangle\langle 0|-| 1\rangle\langle 1|$ is the Pauli operator of the sensor qubit, and $\hat{E}(t)$ is an operator that includes the target signal that oscillates at a particular frequency as well as the presence of noisy environmental fluctuations. An example of sensor qubits is a single NV center, which will be our experimental platform to benchmark sensing protocols. For all experiments in this work, a bias magnetic field between 400 and $500 \mathrm{G}$ aligned with the $\mathrm{NV}$ axis splits the degenerate $m_{s}= \pm 1$ spin states, allowing selective addressing of the $m_{s}=0 \leftrightarrow m_{s}=-1$ transition, which represents our sensor qubit with the qubit states $|0(1)\rangle$ [see Fig. 1(a) and [48] for details of the experimental setup]. In the case of nuclearspin sensing, $\hat{E}(t)$ contains target and bath nuclear-spin operators oscillating at their Larmor frequencies. For ac 


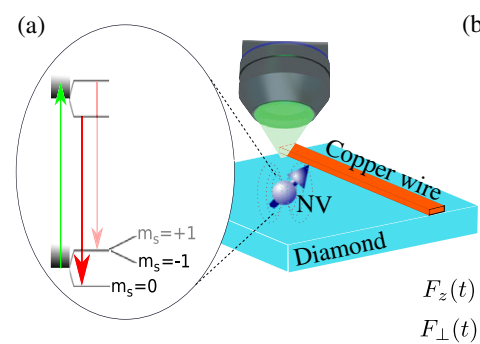

(b)

(c) Standard $\Rightarrow$ repeat $M$ times

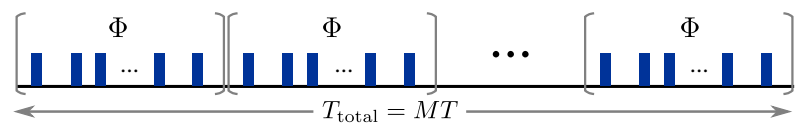

(d) $\quad$ RAND $\Rightarrow\left\{\Phi_{r, 1}, \Phi_{r, 2}, \ldots, \Phi_{r, M}\right\}$

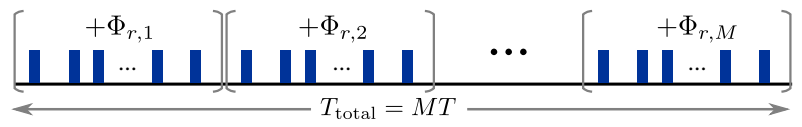

FIG. 1. Randomization protocol for quantum sensing. (a) Experimental setup with an NV center in diamond used as a quantum sensor. (b) Basic unit of pulse sequence for quantum sensing, which is defined by the positions and phases of the $\pi$ pulses. The lower panel is the example of an $X Y 8$ sequence with its associated $F_{z}(t)$ and $F_{\perp}(t)$. (c) Standard way to construct a longer sensing sequence by repeating the same basic pulse unit in Fig. 1(b) $M$ times. (d) Randomization protocol shifting all the pulses within each unit by a common random phase $\Phi_{r, m}$. Random phases $\Phi_{r, m}$ at different blocks are independent. One may refresh all random phases $\left\{\Phi_{r, 1}, \Phi_{r, 2}, \ldots, \Phi_{r, M}\right\}$ at different runs of the sensing experiment.

magnetometry, $\hat{E}(t)$ describes classical oscillating magnetic fields.

The aim of quantum sensing is to detect a target such as a single nuclear spin via the control of the quantum sensor with a sequence of $\mathrm{DD} \pi$ pulses. The latter often corresponds to a periodic repetition of a basic pulse unit that has a time duration $T$ and a number $N$ of pulses [see Fig. 1(b)]. The propagator of a single $\pi$ pulse unit in a general form reads $\hat{U}_{\text {unit }}\left(\left\{\phi_{j}\right\}\right)=\hat{\mathrm{f}}_{N+1} \hat{P}\left(\phi_{N}\right) \hat{\mathrm{f}}_{N} \cdots \hat{P}\left(\phi_{2}\right) \hat{\mathrm{f}}_{2} \hat{P}\left(\phi_{1}\right) \hat{\mathrm{f}}_{1}$, where $\hat{\mathrm{f}}_{j}$ are the free evolutions separated by the control $\pi$ pulses with the propagator $\hat{P}\left(\phi_{j}\right)$. Errors in the control are included in $\hat{P}\left(\phi_{j}\right)$, whereas the different pulse phases $\phi_{j}$ are used by robust DD sequences to mitigate the effect of detuning and amplitude errors of the $\pi$ pulses. Using $M$ repetitions of the basic DD unit [see Fig. 1(c) for the case of a standard construction] allows for an $M$-fold increased signal accumulation time of $T_{\text {total }}=M T$, which enhances the acquired contrast of the weak signal as $\propto M^{2}$ [29] and improves the fundamental frequency resolution to $\sim 1 / T_{\text {total }}$.

To see how a target signal is sensed, we write the Hamiltonian $H^{\prime}(t)$ in the interaction picture of the DD control as [48]

$\hat{H}(t)=\frac{1}{2} F_{z}(t) \hat{\sigma}_{z} \hat{E}(t)+\frac{1}{2}\left[F_{\perp}(t) \hat{\sigma}_{-}+\right.$H.c. $] \hat{E}(t)$, where $\hat{\sigma}_{-}=|0\rangle\langle 1|$. For ideal instantaneous $\pi$ pulses, $F_{\perp}(t)=0$ vanishes [see Fig. 1(b), which shows how the $F_{\perp}(t)$ vanishes between the $\pi$ pulses] and the modulation function $F_{z}(t)$ is the stepped modulation function widely used in the literature; that is, $F_{z}(t)=(-1)^{m}$ when $m \pi$ pulses have been applied up to the moment $t$. The role of a DD-based quantum sensing sequence is to tailor $F_{z}(t)$ such that it oscillates at the same frequency as the target signal in $\hat{E}(t)$, allowing resonant coherent coupling between the sensor and the target.

In realistic situations, where the $\pi$ pulses are not instantaneous due to limited control power, the function $F_{\perp}(t)$ has a nonzero value during $\pi$ pulse execution and $F_{z}(t)$ deviates from \pm 1 [45,53] [see Fig. 1(b) for the example of $X Y 8$ sequences]. Although it is possible to eliminate the effect of deviation in $F_{z}(t)$ by the pulse shaping technique [54], the presence of nonzero $F_{\perp}(t)$ may still alter the expected signal or cause spurious peaks to appear [43]. In general, an oscillating component with a frequency of $k / T_{\text {total }}$ (with $k$ being an integer) in $\hat{E}(t)$, which is not resonant with $F_{z}(t)$, will create a spurious response when the Fourier amplitude $[45,48]$

$$
f_{k}^{\perp}=\frac{1}{T_{\text {total }}} \int_{0}^{T_{\text {total }}} F_{\perp}(t) \exp \left(-i \frac{2 \pi k t}{T_{\text {total }}}\right) d t
$$

of $F_{\perp}(t)$ is nonzero. This spurious response can cause false signal identification (e.g., a wrong conclusion on the detected nuclear species [43]), which is exemplified in Figs. 2 and 3. Suppressing the spurious response from the ${ }^{13} \mathrm{C}$ nuclei is especially critical because it allows reliable nanoscale NMR or MRI without the use of hard to manufacture, and consequently expensive, highly isotopically ${ }^{12} \mathrm{C}$ purified diamond. However, as shown in Figs. 2(c) and 2(d), even for a $Y Y 8$ sequence (designed to remove spurious resonances [46]), the target proton signal is still perturbed by other nuclear species $\left({ }^{13} \mathrm{C}\right.$ in this case). In the presence of amplitude and detuning errors, standard strategies perform even worse.

To remove all spurious peaks, one seeks to design a DD sequence that minimizes the effect of $F_{\perp}(t)$ in a robust manner. We observe that, by introducing a global phase to all the $\pi$ pulses, the form of $F_{z}(t)$ is unchanged but a phase factor is added to $F_{\perp}(t)$. This motivates the following method to preserve $F_{z}(t)$ and to suppress the effect of $F_{\perp}(t)$ by phase randomization.

Phase randomization.-In the randomization protocol, a random global phase $\Phi_{r, m}$ (where the subscript $r$ means a random value) is added to all the pulses within each unit $m$, as shown in Fig. 1(d). The propagator of $M$ DD units with independent global phases reads $\hat{U}_{r}=$ $\prod_{m=1}^{M} \hat{U}_{\text {unit }}\left(\left\{\phi_{j}+\Phi_{r, m}\right\}\right)$. If one sets all the random phases $\Phi_{r, m}$ to the same value (e.g., zero), the original DD sequence can be recovered [Fig. 1(c)]. Because each of 


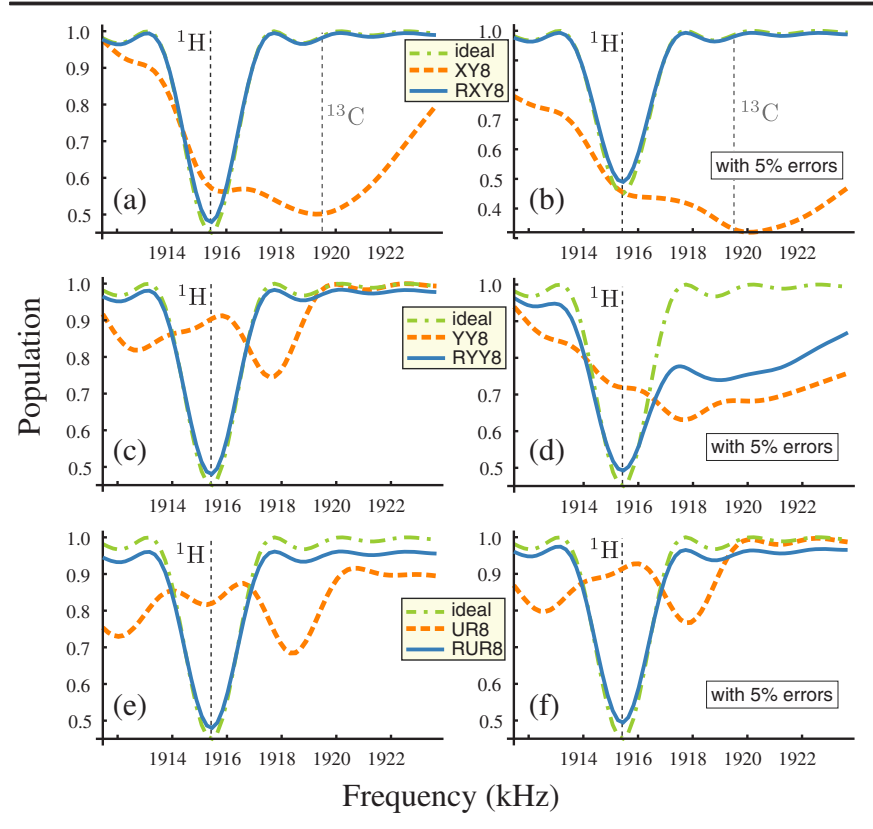

FIG. 2. Quantum spectroscopy with DD. (a) Simulated averaged population signal as a function of the DD frequency $[1 /(2 \tau)$ for pulse spacing $\tau$ ]. One ${ }^{1} \mathrm{H}$ spin and one ${ }^{13} \mathrm{C}$ spin are coupled to the $\mathrm{NV}$ center via the hyperfine-field components $[41,48]\left(A_{\perp}, A_{\|}\right)=$ $2 \pi \times(2,1) \mathrm{kHz}$ and $2 \pi \times(5,50) \mathrm{kHz}$, respectively. The orange dashed line (blue solid line) is the signal obtained by a standard $X Y 8$ (randomized $X Y 8$ ) sequence using rectangular $\pi$ pulses with a time duration of $200 \mathrm{~ns}$ and $M=200$. The presence of the ${ }^{13} \mathrm{C}$ distorts the proton spin signal centered at the proton spin frequency (see the vertical dashed lines indicating the target ${ }^{1} \mathrm{H}$ and the spurious ${ }^{13} \mathrm{C}$ resonance frequencies for a magnetic field of $450 \mathrm{G}$ ). The randomized $X Y 8$ sequence significantly reduces the signal distortion due to noninstantaneous control and reveals the real proton signal (see the green dash-dotted line for the signal obtained by a perfect $X Y 8$ sequence). (b) Like Fig. 2(a) but adding 5\% (in terms of the ideal Rabi frequency) of errors in both driving amplitude and frequency detuning to the $\pi$ pulses. (c) and (d) [(e) and (f)] Same as Figs. 2(a) and 2(b) but for the $Y Y 8$ [46] and randomized $Y Y 8(R Y Y 8)$ [UR8 [42] and randomized UR8 (RUR8)] sequences. Despite the $Y Y 8$ sequence-which uses single-axis control to mitigate the spurious peak in the ${ }^{13} \mathrm{C}$ spectrum when there is no pulse error- the presence of the ${ }^{13} \mathrm{C}$ still distorts the proton spin signal centered at the proton spin frequency. In all cases, the randomization protocol reduces the signal distortion due to noninstantaneous control and control errors.

the global phases does not change the internal structure (i.e., the relative phases among $\pi$ pulses) of the basic unit, the robustness of the basic DD sequence is preserved. On the other hand, as we will show in the following, these random global phases prevent control imperfections from accumulating.

Universal suppression of spurious response.-The randomization protocol provides a universal method to suppress spurious responses. For the sequence with randomization, one can find that the Fourier amplitude reads $f_{k}^{\perp}=Z_{r, M} \tilde{f}_{k / M}^{\perp}$, where

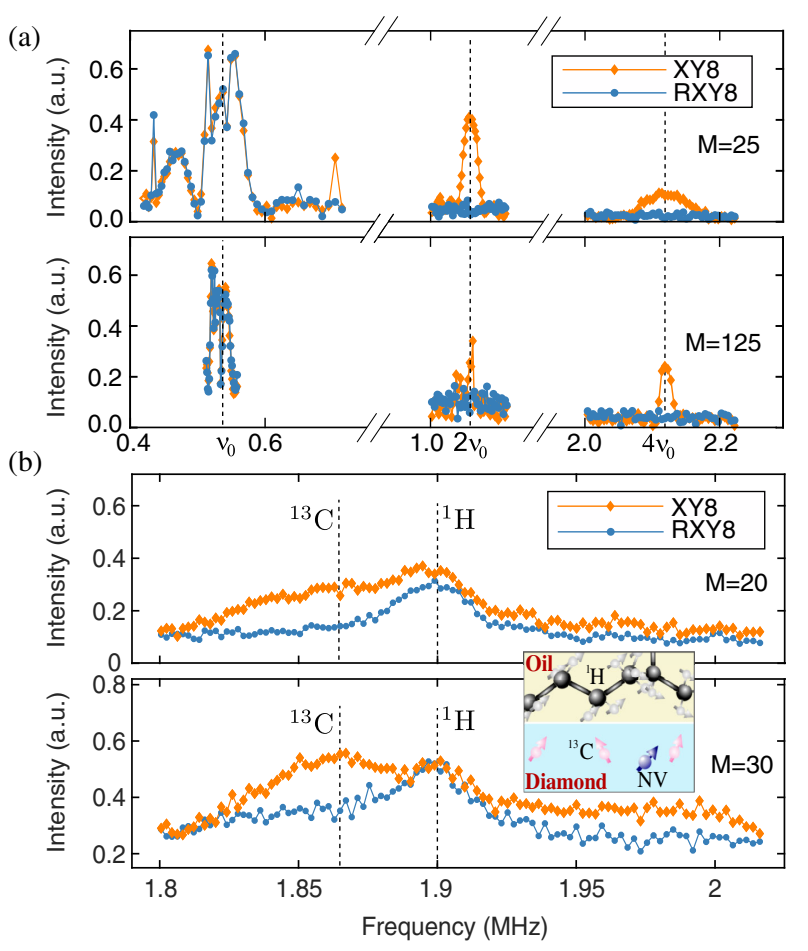

FIG. 3. Removing spurious response with the phase randomization protocol. (a) In the measured spectrum of an ac magnetic field sensed by a standard repetition of the $X Y 8$ sequence (see orange diamonds), the noninstantaneous $\pi$ pulses produce spurious peaks at frequencies $2 \nu_{0}$ and $4 \nu_{0}$. Repeating the $X Y 8$ sequence with phase randomization (see blue bullets) preserves the desired signal centered at $\nu_{0}$ and efficiently suppresses all the spurious peaks. The $X Y 8$ unit was repeated $M=25$ times in the upper panel and $M=125$ in the lower panel for a longer sensing time. Here, the ac field was generated by a signal generator and the diamond was isotopically enriched to remove ${ }^{13} \mathrm{C}$ spins [48]. (b) Detection of proton spins in the immersion oil (Fluka Analytical 10976) located on the surface of the diamond using the $X Y 8$ sequence (see inset). Different from the case in Fig. 3(a), here, we used a natural ${ }^{13} \mathrm{C}$ abundance diamond and a shallow NV within it [48]. For the measured spectrum obtained by the standard protocol, the ${ }^{13} \mathrm{C}$ nuclear spins produce a strong and wide spurious peak that hinders proton spin detection. Using the randomization protocol, the spurious ${ }^{13} \mathrm{C}$ peak has been suppressed, revealing the proton spin signal centered around a frequency of $1.9 \mathrm{MHz}$.

$$
\tilde{f}_{k / M}^{\perp}=\frac{1}{T} \int_{0}^{T} F_{\perp}(t) \exp \left(-i \frac{2 \pi k t}{M T}\right) d t
$$

is the Fourier component defined over a single period $T$ [48]. For random phases $\left\{\Phi_{r, m}\right\}$, the factor

$$
Z_{r, M}=\frac{1}{M} \sum_{m=1}^{M} \exp \left(i \Phi_{r, m}\right)
$$

captures the effect of the randomization protocol. Due to the random values of the phases $\Phi_{r, m}, Z_{r, M}$ becomes a 
(normalized) 2D random walk with $\left\langle\left|Z_{r, M}\right|^{2}\right\rangle=1 / M$, thus suppressing the contrast of a spurious response by a factor of $1 /(2 M)$ as compared with the standard protocol [48]. Here, we note that one can design a set of specific (i.e., not random) phases $\Phi_{r, m}$ that minimizes a certain $f_{k}^{\perp}$ completely. However, this set of phases would be specific to one $k$ value (i.e., it does not suppress all spurious peaks simultaneously). In this respect, the power of our method is that it is simple to implement and fully universal, suppressing all spurious peaks produced by any sequence while still retaining the ideal signal, as shown in Fig. 2.

To experimentally benchmark the performance, we carried out nanoscale detection of a classical ac magnetic field generated by a signal generator [Fig. 3(a)] and, separately, the nanoscale NMR detection of an ensemble of proton spins in a drop of immersion oil (Fluka Analytical 10976) in direct contact with a natural ${ }^{13} \mathrm{C}$ abundance (1.1\%) diamond [Fig. 3(b)]. The standard repetition of the $X Y 8$ sequence, which was widely used in various sensing and sensing-based applications (e.g., see Refs. [11-14, $17-19,33,38,43]$ ), produces spurious peaks when the duration of $\pi$ pulses is nonzero. In contrast, the randomization protocol suppresses all the spurious peaks in the spectrum efficiently, and the spurious background noise from a ${ }^{13} \mathrm{C}$ nuclear-spin bath in diamond was removed while the desired proton signal was unaffected, demonstrating a clear and unambiguous proton spin detection without the use of hard to manufacture, expensive, ${ }^{12} \mathrm{C}$ isotopically pure diamonds.

In the experiments, we have repeated the randomization protocol with $K=10$ samples of the random phase sequences $\left\{\Phi_{r, m}\right\}$ and averaged out the measured signals. This reduces the fluctuations of the (suppressed) spurious peaks introduced by the applied random phases because the variance of $\left|Z_{r, M}\right|^{2}$ [which is $(M-1) / M^{3}$ ] is further reduced by a factor of $1 / K$ [48].

Removing the spurious response also improves the accuracy, for example, in measuring the depth of individual NV centers [19]. By falsely assuming that all the signal around $1.9 \mathrm{MHz}$ obtained by the standard XY8 sequences originates from hydrogen spins in the immersion oil, the computed NV center depth would be $5.88 \pm 0.52 \mathrm{~nm}$ instead of the $7.62 \pm 0.29 \mathrm{~nm}$ obtained by the randomized $X Y 8$ - a deviation of about 30\% [see Fig. 3(b)].

Enhancement on control robustness.-As indicated in Fig. 2, the randomization protocol also enhances the robustness of the whole DD sequence. For simplicity, in the following discussion, we neglect the effect of the environment and concentrate on static control imperfections. The latter introduce errors in the form of nonzero matrix elements $\left\langle 0\left|\hat{U}_{\text {unit }}\right| 1\right\rangle=C \epsilon+O\left(\epsilon^{2}\right)$, where $\epsilon$ is a small parameter and $C$ is a prefactor depending on the explicit form of control (see [48] for details). For the standard protocol where the same $\hat{U}_{\text {unit }}$ block is repeated, the static errors accumulate coherently, yielding $\quad\left\langle 0\left|\left(\hat{U}_{\text {unit }}\right)^{M}\right| 1\right\rangle=M C \epsilon+O\left(\epsilon^{2}\right)$. The random phases in the randomization protocol avoid this coherent error accumulation, and one can find $\left\langle 0\left|\hat{U}_{r}\right| 1\right\rangle=Z_{r, M} M C \epsilon+O\left(\epsilon^{2}\right)$, where the error is suppressed by the factor $Z_{r, M}$, which is given by Eq. (3) for random phases [48]. Compared with the suppression of control imperfections by deterministic phases, the randomization protocol is universal and achieves both suppression of the spurious response and enhancement of robustness, without loss of sensitivity to target signals, as shown in Figs. 2 and 3.

In Fig. 4(a), we show the robustness of the widely used $X Y 8$ sequence, with respect to the amplitude bias and frequency detuning of the microwave pulses, for the randomization and standard protocols. The simulation and experiment demonstrate robustness improvement after applying phase randomization.

Robust DD protocols typically employ rotations about different axes. For example, the $X Y 8$ unit consists of eight $\pi$ pulses about the $x$ and $y$ axes, which have a phase difference of $\pi / 2$ [see Fig. 1(b)]. As shown in Fig. 4(b), the randomization protocol also suppresses errors in these pulse phases. The latter is especially relevant for digital pulsing devices where the signal from a microwave source is split up and the phase in one arm is shifted by suitable equipment. On top of errors due to the working accuracy of these devices, different cable lengths in both arms can sum up to errors in the relative phase.
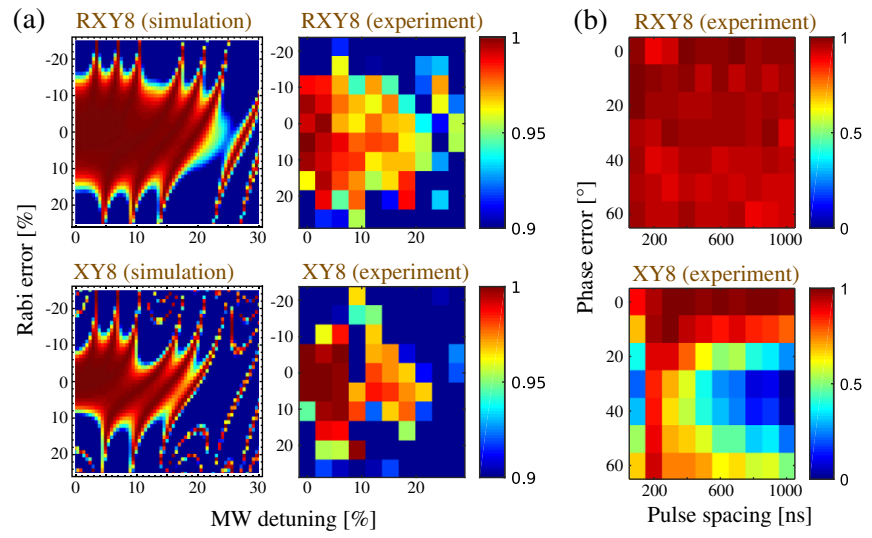

FIG. 4. Experimental enhancement of sequence robustness with the phase randomization protocol. (a) Fidelity of $X Y 8$ sequences as a function of detuning and Rabi frequency errors for randomization (upper panels) and standard (lower panels) protocols. The control errors are measured in terms of the ideal Rabi frequency $\Omega_{\text {ideal }}=2 \pi \times 32.8 \mathrm{MHz}$. The sequences have interpulse spacing of $200 \mathrm{~ns}$ and $M=25 X Y 8$ units. (b) Fidelity of $X Y 8$ sequences with respect to a static phase error between the $X$ and $Y$ pulses and the interpulse time interval $\tau$ for randomization (upper panels) and standard (lower panels) protocols with $M=12$. Resonant microwave $\pi$ pulses are used with a Rabi frequency of $\Omega_{\text {ideal }}=2 \pi \times 66.6 \mathrm{MHz}$. Both in Figs. 4(a) and 4(b), the diamond is the same as that used in Fig. 3(a) [48]. 
Conclusion.-We present a randomization protocol for DD sequences that efficiently and universally suppress a spurious response while maintaining the expected signal. This method is simple to implement, only requiring additional random control-pulse phases, and is valid for all DD sequence choices. The protocol functions equally well for quantum and classical signals, allowing clear and unambiguous ac field and nuclear-spin detection, e.g., with the widely used $X Y$ family of sequences. Furthermore, the protocol also enhances the robustness of whole pulse sequences. For sensing experiments with NV centers, the protocol reduces the reliance on hard to manufacture, expensive, highly isotopically purified diamond. The method has a general character, being equally applicable to other quantum platforms and other DD applications. For example, it could be used to improve correlation spectroscopy $[18,32,55,56]$ in quantum sensing and fast quantum gates in trapped ions $[57,58]$ where DD has been used as an important ingredient.

M. B. P. and Z.-Y. W. acknowledge support by the ERC Synergy grant BioQ (Grant No. 319130), the EU projects HYPERDIAMOND and AsteriQs, the QuantERA project NanoSpin, the BMBF project DiaPol, the state of BadenWürttemberg through the bwHPC, and the German Research Foundation (DFG) through Grant No. INST 40/467-1 FUGG. J.E. L. is funded by an EPSRC Doctoral Prize Fellowship. F. J., S. S., L. M., and J. L. acknowledge the support of Q-Magine of the QUANTERA, DFG (FOR 1493, SPP 1923, JE 290/18-1, and SFB 1279), BMBF (13N14438, 16KIS0832, and 13N14810), ERC (BioQ 319130), VW Stiftung, and Landesstiftung BW. J. C. acknowledges financial support from Juan de la Cierva Grant No. IJCI-2016-29681 and Basque Government Grant IT986-16.

Z.-Y. W., J.E. L, and S.S. contributed equally to this work.

*zhenyu.wang@uni-ulm.de

[1] M. W. Doherty, N. B. Manson, P. Delaney, F. Jelezko, J. Wrachtrup, and L. C. L. Hollenberg, The nitrogen-vacancy colour centre in diamond, Phys. Rep. 528, 1 (2013).

[2] R. Schirhagl, K. Chang, M. Loretz, and C. L. Degen, Nitrogen-Vacancy Centers in Diamond: Nanoscale Sensors for Physics and Biology, Annu. Rev. Phys. Chem. 65, 83 (2014).

[3] L. Rondin, J. P. Tetienne, T. Hingant, J. F. Roch, P. Maletinsky, and V. Jacques, Magnetometry with nitrogen-vacancy defects in diamond, Rep. Prog. Phys. 77, 056503 (2014).

[4] D. Suter and F. Jelezko, Single-spin magnetic resonance in the nitrogen-vacancy center of diamond, Prog. Nucl. Magn. Reson. Spectrosc. 98-99, 50 (2017).

[5] Y. Wu, F. Jelezko, M. B. Plenio, and T. Weil, Diamond Quantum Devices in Biology, Angew. Chem. Int. Ed. 55, 6586 (2016).
[6] A. M. Souza, G. A. Álvarez, and D. Suter, Robust dynamical coupling, Phil. Trans. R. Soc. A 370, 4748 (2012).

[7] C. A. Ryan, J.S. Hodges, and D. G. Cory, Robust Decoupling Techniques to Extend Quantum Coherence in Diamond, Phys. Rev. Lett. 105, 200402 (2010).

[8] G. de Lange, Z. H. Wang, D. Ristè, V. V. Dobrovitski, and R. Hanson, Universal dynamical decoupling of a single solid-state spin from a spin bath, Science 330, 60 (2010).

[9] B. Naydenov, F. Dolde, L. T. Hall, C. Shin, H. Fedder, L. C. L. Hollenberg, F. Jelezko, and J. Wrachtrup, Dynamical Decoupling of a Single-Electron Spin at Room Temperature, Phys. Rev. B 83, 081201(R) (2011).

[10] G. de Lange, D. Ristè, V. V. Dobrovitski, and R. Hanson, Single-Spin Magnetometry with Multipulse Sensing Sequences, Phys. Rev. Lett. 106, 080802 (2011).

[11] T. Staudacher, F. Shi, S. Pezzagna, J. Meijer, J. Du, C. A. Meriles, F. Reinhard, and J. Wrachtrup, Nuclear Magnetic Resonance Spectroscopy on a (5-Nanometer) ${ }^{3}$ Sample Volume, Science 339, 561 (2013).

[12] D. Rugar, H. J. Mamin, M. H. Sherwood, M. Kim, C. T. Rettner, K. Ohno, and D. D. Awschalom, Proton magnetic resonance imaging using a nitrogen-vacancy spin sensor, Nat. Nanotechnol. 10, 120 (2015).

[13] S. J. DeVience, L. M. Pham, I. Lovchinsky, A. O. Sushkov, N. Bar-Gill, C. Belthangady, F. Casola, M. Corbett, H. Zhang, M. Lukin, H. Park, A. Yacoby, and R. L. Walsworth, Nanoscale NMR spectroscopy and imaging of multiple nuclear species, Nat. Nanotechnol. 10, 129 (2015).

[14] F. Shi, Q. Zhang, P. Wang, H. Sun, J. Wang, X. Rong, M. Chen, C. Ju, F. Reinhard, H. Chen, J. Wrachtrup, J. Wang, and J. Du, Single-protein spin resonance spectroscopy under ambient conditions, Science 347, 1135 (2015).

[15] S. Schmitt, T. Gefen, F. M. Stürner, T. Unden, G. Wolff, C. Müller, J. Scheuer, B. Naydenov, M. Markham, S. Pezzagna, J. Meijer, I. Schwarz, M. Plenio, A. Retzker, L. P. McGuinness, and F. Jelezko, Submillihertz magnetic spectroscopy performed with a nanoscale quantum sensor, Science 356, 832 (2017).

[16] J. M. Boss, K. S. Cujia, J. Zopes, and C. L. Degen, Quantum sensing with arbitrary frequency resolution, Science 356, 837 (2017).

[17] D. R. Glenn, D. B. Bucher, J. Lee, M. D. Lukin, H. Park, and R. L. Walsworth, High-resolution magnetic resonance spectroscopy using a solid-state spin sensor, Nature (London) 555, 351 (2018).

[18] T. Rosskopf, J. Zopes, J. M. Boss, and C. L. Degen, A quantum spectrum analyzer enhanced by a nuclear-spin memory, npj Quantum Inf. 3, 33 (2017).

[19] L. M. Pham, S. J. DeVience, F. Casola, I. Lovchinsky, A. O. Sushkov, E. Bersin, J. Lee, E. Urbach, P. Cappellaro, H. Park, A. Yacoby, M. Lukin, and R. L. Walsworth, NMR technique for determining the depth of shallow nitrogen-vacancy centers in diamond, Phys. Rev. B 93, 045425 (2016).

[20] T. H. Taminiau, J. J. T. Wagenaar, T. van der Sar, F. Jelezko, V. V. Dobrovitski, and R. Hanson, Detection and Control of Individual Nuclear Spins using a Weakly Coupled Electron Spin, Phys. Rev. Lett. 109, 137602 (2012).

[21] S. Kolkowitz, Q. P. Unterreithmeier, S. D. Bennett, and M. D. Lukin, Sensing Distant Nuclear Spins with a Single Electron Spin, Phys. Rev. Lett. 109, 137601 (2012). 
[22] N. Zhao, J. Honert, B. Schmid, M. Klas, J. Isoya, M. Markham, D. Twitchen, F. Jelezko, R. B. Liu, H. Fedder, and J. Wrachtrup, Sensing single remote nuclear spins, Nat. Nanotechnol. 7, 657 (2012).

[23] C. Müller, X. Kong, J.-M. Cai, K. Melentijević, A. Stacey, M. Markham, D. Twitchen, J. Isoya, S. Pezzagna, J. Meijer, J. Du, M. B. Plenio, B. Naydenov, L. P. McGuinness, and F. Jelezko, Nuclear magnetic resonance spectroscopy with single spin sensitivity, Nat. Commun. 5, 4703 (2014).

[24] J.E. Lang, R. B. Liu, and T. S. Monteiro, DynamicalDecoupling-Based Quantum Sensing: Floquet Spectroscopy, Phys. Rev. X 5, 041016 (2015).

[25] K. Sasaki, K. M. Itoh, and E. Abe, Determination of the position of a single nuclear spin from free nuclear precessions detected by a solid-state quantum sensor, Phys. Rev. B 98, 121405(R) (2018).

[26] J. Zopes, K. S. Cujia, K. Sasaki, J. M. Boss, K. M. Itoh, and C. L. Degen, Three-dimensional localization spectroscopy of individual nuclear spins with sub-Angstrom resolution, Nat. Commun. 9, 4678 (2018).

[27] J. Zopes, K. Herb, K. S. Cujia, and C. L. Degen, ThreeDimensional Nuclear Spin Positioning Using Coherent Radio-Frequency Control, Phys. Rev. Lett. 121, 170801 (2018).

[28] M. Pfender, P. Wang, H. Sumiya, S. Onoda, W. Yang, D. B. R. Dasari, P. Neumann, X.-Y. Pan, J. Isoya, R.-B. Liu, and J. Wrachtrup, High-resolution spectroscopy of single nuclear spins via sequential weak measurements, Nat. Commun. 10, 594 (2019).

[29] N. Zhao, J.-L. Hu, S.-W. Ho, J. T. K. Wan, and R. B. Liu, Atomic-scale magnetometry of distant nuclear spin clusters via nitrogen-vacancy spin in diamond, Nat. Nanotechnol. 6, 242 (2011).

[30] F. Shi, X. Kong, P. Wang, F. Kong, N. Zhao, R.-B. Liu, and J. Du, Sensing and atomic-scale structure analysis of single nuclear-spin clusters in diamond, Nat. Phys. 10, 21 (2014).

[31] Z.-Y. Wang, J. F. Haase, J. Casanova, and M. B. Plenio, Positioning nuclear spins in interacting clusters for quantum technologies and bioimaging, Phys. Rev. B 93, 174104 (2016).

[32] Z.-Y. Wang, J. Casanova, and M. B. Plenio, Delayed entanglement echo for individual control of a large number of nuclear spins, Nat. Commun. 8, 14660 (2017).

[33] M. H. Abobeih, J. Cramer, M. A. Bakker, N. Kalb, M. Markham, D. J. Twitchen, and T. H. Taminiau, One-second coherence for a single electron spin coupled to a multi-qubit nuclear-spin environment, Nat. Commun. 9, 2552 (2018).

[34] C. L. Degen, F. Reinhard, and P. Cappellaro, Quantum sensing, Rev. Mod. Phys. 89, 035002 (2017).

[35] J. Casanova, Z.-Y. Wang, and M. B. Plenio, Noise-Resilient Quantum Computing with a Nitrogen-Vacancy Center and Nuclear Spins, Phys. Rev. Lett. 117, 130502 (2016).

[36] J. Casanova, Z.-Y. Wang, and M. B. Plenio, Arbitrary nuclearspin gates in diamond mediated by a nitrogen-vacancy-center electron spin, Phys. Rev. A 96, 032314 (2017).

[37] J. Cai, A. Retzker, F. Jelezko, and M. B. Plenio, A largescale quantum simulator on a diamond surface at room temperature, Nat. Phys. 9, 168 (2013).

[38] P. C. Humphreys, N. Kalb, J. P. J. Morits, R. N. Schouten, R. F. L. Vermeulen, D. J. Twitchen, M. Markham, and
R. Hanson, Deterministic delivery of remote entanglement on a quantum network, Nature (London) 558, 268 (2018).

[39] M. A. Perlin, Z.-Y. Wang, J. Casanova, and M. B. Plenio, Noise-resilient architecture of a hybrid electron-nuclear quantum register in diamond, Quantum Sci. Technol. 4, 015007 (2019).

[40] T. Gullion, D. B. Barker, and M. S. Conradi, New, compensated Carr-Purcell sequences, J. Magn. Reson. 89, 479 (1990).

[41] J. Casanova, Z.-Y. Wang, J. F. Haase, and M. B. Plenio, Robust dynamical decoupling sequences for individualnuclear-spin addressing, Phys. Rev. A 92, 042304 (2015).

[42] G. T. Genov, D. Schraft, N. V. Vitanov, and T. Halfmann, Arbitrarily Accurate Pulse Sequences for Robust Dynamical Decoupling, Phys. Rev. Lett. 118, 133202 (2017).

[43] M. Loretz, J. M. Boss, T. Rosskopf, H. J. Mamin, D. Rugar, and C. L. Degen, Spurious Harmonic Response of Multipulse Quantum Sensing Sequences, Phys. Rev. X 5, 021009 (2015).

[44] J. F. Haase, Z.-Y. Wang, J. Casanova, and M. B. Plenio, Pulse-phase control for spectral disambiguation in quantum sensing protocols, Phys. Rev. A 94, 032322 (2016).

[45] J. E. Lang, J. Casanova, Z.-Y. Wang, M. B. Plenio, and T. S. Monteiro, Enhanced Resolution in Nanoscale NMR via Quantum Sensing with Pulses of Finite Duration, Phys. Rev. Applied 7, 054009 (2017).

[46] Z. Shu, Z. Zhang, Q. Cao, P. Yang, M. B. Plenio, C. Müller, J. Lang, N. Tomek, B. Naydenov, L. P. McGuinness, F. Jelezko, and J. Cai, Unambiguous nuclear spin detection using an engineered quantum sensing sequence, Phys. Rev. A 96, 051402(R) (2017).

[47] J. F. Haase, Z.-Y. Wang, J. Casanova, and M. B. Plenio, Soft Quantum Control for Highly Selective Interactions among Joint Quantum Systems, Phys. Rev. Lett. 121, 050402 (2018).

[48] See Supplemental Material at http://link.aps.org/ supplemental/10.1103/PhysRevLett.122.200403 for more details, which includes Refs. [49-52].

[49] C. Osterkamp, J. Lang, J. Scharpf, C. Müller, L. P. McGuinness, T. Diemant, R. J. Behm, B. Naydenov, and F. Jelezko, Stabilizing shallow color centers in diamond created by nitrogen delta-doping using $\mathrm{SF}_{6}$ plasma treatment, Appl. Phys. Lett. 106, 113109 (2015).

[50] P. Fernández-Acebal, O. Rosolio, J. Scheuer, C. Müller, S. Müller, S. Schmitt, L. P. McGuinness, I. Schwarz, Q. Chen, A. Retzker, B. Naydenov, F. Jelezko, and M. B. Plenio, Toward Hyperpolarization of Oil Molecules via Single Nitrogen Vacancy Centers in Diamond, Nano Lett. 18, 1882 (2018).

[51] J. M. Binder, A. Stark, N. Tomek, J. Scheuer, F. Frank, K. D. Jahnke, C. Müller, S. Schmitt, M. H. Metsch, T. Unden, T. Gehring, A. Huck, U. L. Andersen, L. J. Rogers, and F. Jelezko, Qudi: A modular python suite for experiment control and data processing, SoftwareX 6, 85 (2017).

[52] H. Y. Carr and E. M. Purcell, Effects of diffusion on free precession in nuclear magnetic resonance experiments, Phys. Rev. 94, 630 (1954).

[53] J. E. Lang, T. Madhavan, J.-P. Tetienne, D. A. Broadway, L. T. Hall, T. Teraji, T. S. Monteiro, A. Stacey, and L. C. L. Hollenberg, Nonvanishing effect of detuning errors in dynamical-decoupling-based quantum sensing experiments, Phys. Rev. A 99, 012110 (2019). 
[54] J. Casanova, Z.-Y. Wang, I. Schwartz, and M. B. Plenio, Shaped Pulses for Energy-Efficient High-Field NMR at the Nanoscale, Phys. Rev. Applied 10, 044072 (2018).

[55] A. Laraoui, F. Dolde, C. Burk, F. Reinhard, J. Wrachtrup, and C. A. Meriles, High-resolution correlation spectroscopy of ${ }^{13} \mathrm{C}$ spins near a nitrogen-vacancy centre in diamond, Nat. Commun. 4, 1651 (2013).

[56] W.-L. Ma and R.-B. Liu, Proposal for Quantum Sensing Based on Two-Dimensional Dynamical Decoupling: NMR
Correlation Spectroscopy of Single Molecules, Phys. Rev. Applied 6, 054012 (2016).

[57] I. Arrazola, J. Casanova, J. S. Pedernales, Z.-Y. Wang, E. Solano, and M. B. Plenio, Pulsed dynamical decoupling for fast and robust two-qubit gates on trapped ions, Phys. Rev. A 97, 052312 (2018).

[58] T. Manovitz, A. Rotem, R. Shaniv, I. Cohen, Y. Shapira, N. Akerman, A. Retzker, and R. Ozeri, Fast Dynamical Decoupling of the Mølmer-Sørensen Entangling Gate, Phys. Rev. Lett. 119, 220505 (2017). 